\title{
Functionally Atrialized Parchment-Like Right Ventricle with Extensive Myocardial Fibrosis of Left Ventricle
}

\author{
Kazuhiko Murata, M.D., Hideyo Matsuo, M.D., Yoshiyuki \\ Yoshitake, M.D., Yoshiharu Ishizawa, M.D., Ryoichi \\ ShigiYa, M.D.,* and Koichi Oкamoto, M.D.
}

\begin{abstract}
SUMMARY
A 18-year-old female with unusual type of parchment-like right ventricle died of intractable congestive heart failure was reported. A catheter study revealed the absence of systolic contraction of the right ventricle. The pressure tracing curve in the right ventricle was virtually the same as in the right atrium. At autopsy, there was an extensive myocardial fibrosis of the left ventricle in addition to the almost total absence of the myocardium of the right ventricle. The case was considered to be a unique type of idiopathic cardiomyopathy.
\end{abstract}

\section{Additional Indexing Words:}

Idiopathic cardiomyopathy Uhl's anomaly

\begin{abstract}
A parchment-like right ventricle, an almost total absence of the myocarA dium of the right ventricle, is a rare abnormality of the heart first described by Uhl in 1952." There are at least 34 reported cases in the world literature thereafter as far as we know. Ostermeyer ${ }^{2)}$ listed 33 reported cases in 1973, while Tomita and associates ${ }^{3)}$ reported an additional case in Japanese. The pathological changes of the heart in this abnormality were usually restricted to the right ventricle in hitherto reported cases, and the involvement of the left ventricle was exceptional. The present paper deals with a unique case of parchment-like right ventricle associated with extensive myocardial fibrosis of both ventricles.
\end{abstract}

\section{CASE REPORT}

K. M., a 18-year-old girl was admitted to the hospital with the chief complaints of exertional dyspnea, palpitation, and pitting edema. The patient was free from symptoms until 1 year before admission, although she was told to have an enlarged

From the Second Department of Internal Medicine and the First Department of Pathology, Gunma University School of Medicine, Maebashi 371, Japan.

* Present address: National Yokohama Hospital, Yokohama.

Received for publication July 15, 1975. 


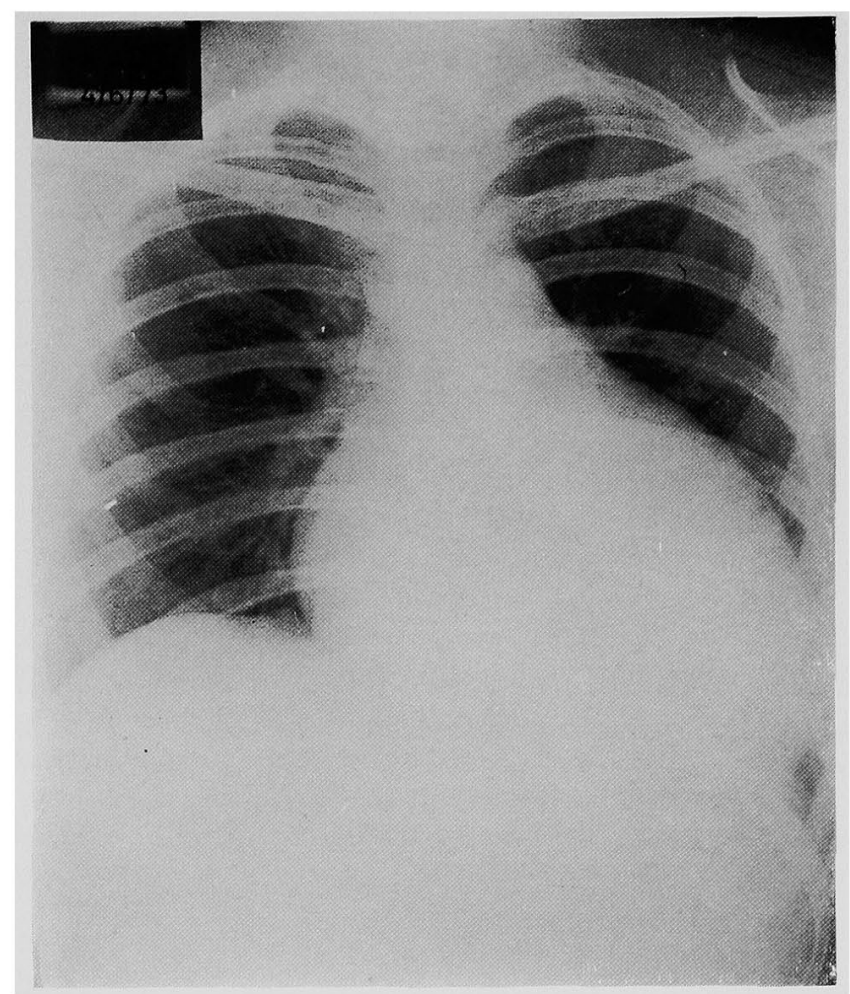

Fig. 1. Chest roentgenogram on admission showing marked enlargement of cardiac silhouette.

heart 2 years ago at annual examination at the school. She noticed palpitation, exertional dyspnea, anorexia, lassitude, and pretibial edema 1 year before admission. The family history was not contributory. The patient's twin sister was entirely healthy. There was no history suggestive of rheumatic fever nor myocarditis.

Physical examination revealed a well developed noncyanotic girl of moderately ill. The heart rate was 105 and regular. The cardiac dullness was endlarged to the left anterior axillary line. The apex beat was not palpable. The cardiac sounds were faint, but no cardiac murmur was heard. There was no gallop rhythm. The lungs were clear and no rales were heard. The liver was palpable 2 fingerbreadths below the right costal margin. The spleen and the kidney were not palpable. There was slight pitting edema of the pretibial region. The blood pressure was $110 / 90 \mathrm{mmHg}$ and the venous pressure was $270 \mathrm{~mm}$ of saline. The hemogram was within normal limits. The urinalysis was negative except for one plus test for albumin. Chemical studies of the blood were within normal limits.

The chest X-ray film showed a marked globular enlargement of cardiac silhouette as seen in Fig. 1. The cardiothoracic ratio was $62 \%$. The pulmonary vascular markings were not striking. The electrocardiogram showed sinus tachycardia at a rate of 105 and right axis deviation. The amplitudes of the QRS waves were low and there were nonspecific changes of $T$ waves in limb and precordial leads as seen in Fig. 2. 


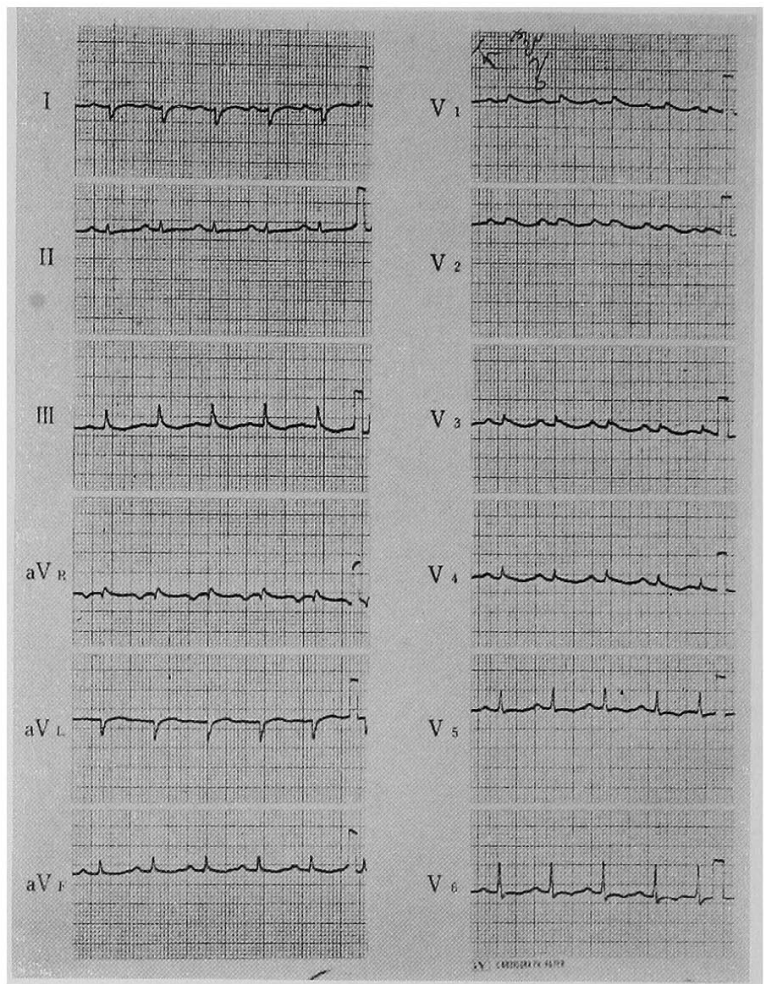

Fig. 2. Electrocardiogram recorded on admission (June 4, 1973) showed sinus tachycardia, right axis deviation, low voltage in limb and precordial leads and nonspecific ST-T change.

Right heart catheterization was performed 2 months after the admission. Although the catheter was introduced into the right ventricle, the pulmonary artery could not be entered in spite of several attempts. The right atrial pressure was markedly elevated. The mean right atrial pressure was $14 \mathrm{mmHg}$. The pattern of the pressure tracing curve in the right ventricle was virtually the same as in the right atrium as seen in Fig. 3. A physiological evidence of the systolic contraction of the right ventricle was not obvious as seen in this figure, and there was no pressure gradient between the right ventricle and the right atrium both in diastole and in systole. The right ventriculography revealed markedly dilated right atrium and right ventricle. The tricuspid valve appeared to be normally situated. The trabeculation of the right ventricle was not obvious and there was a prolonged opacification of the right ventricle.

Although the patient was given oxygen, digitalis and diuretics, the symptoms worsened progressively. Frequent supraventricular premature beats appeared later and the patient died of intractable congestive heart failure 4 months after the admission.

At autopsy (No. 4308), the heart was enormously dilated, particularly the right atrium and the right ventricle. The heart weight was $400 \mathrm{Gm}$. The systemic and pulmonary great vessels were normally situated. The coronary arteries were normal. 


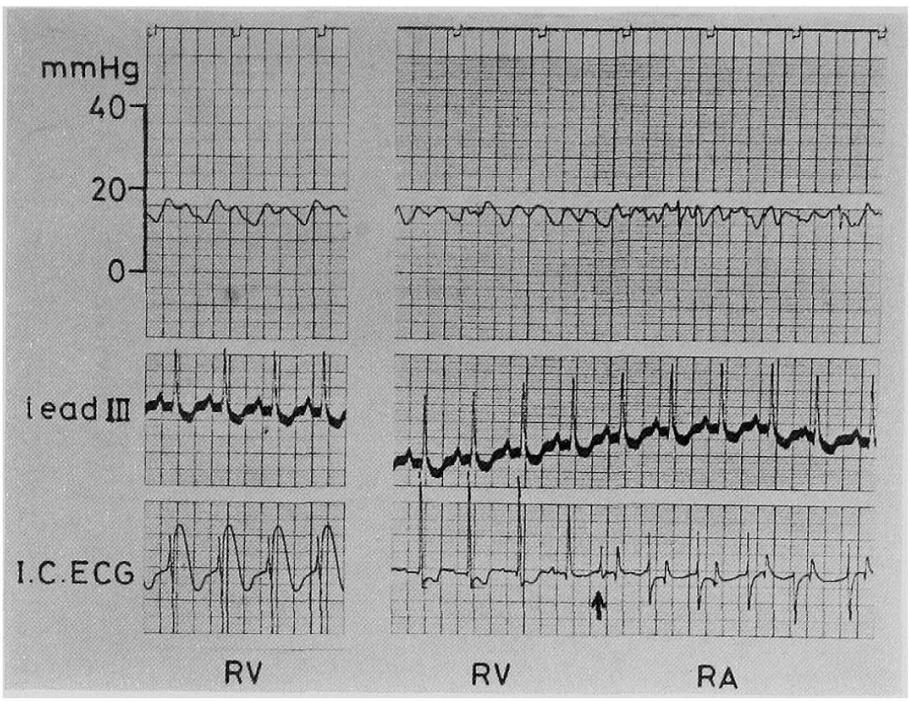

Fig. 3. Pressure tracing curves in right ventricle and right atrium.

The arrow shows the withdrawal of the catheter from the right ventricle to the right atrium. No apparent change of the pressure tracing curve was observed when the catheter was withdrawn. (I.C. ECG: Intracavitary electrocardiogram).

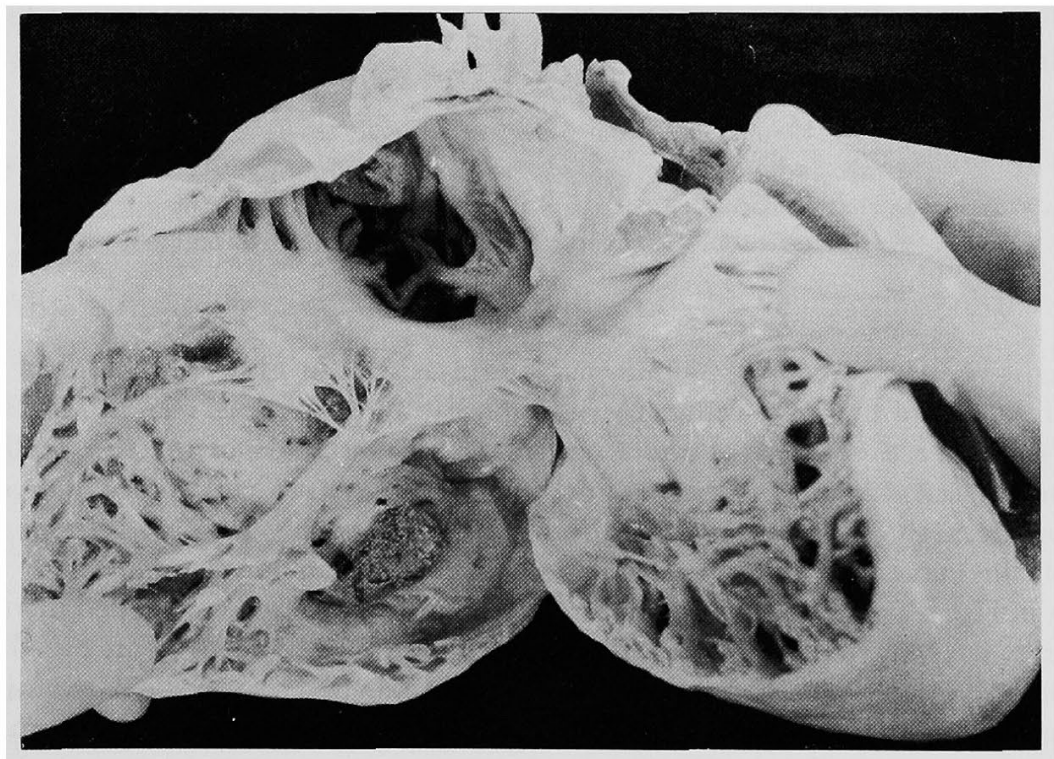

Fig. 4. Opened right atrium and right ventricle.

Both chambers are enormously dilated and the free wall of the right ventricle is paper thin. Large mural thrombi are present in the right atrium and in the right ventricle. 


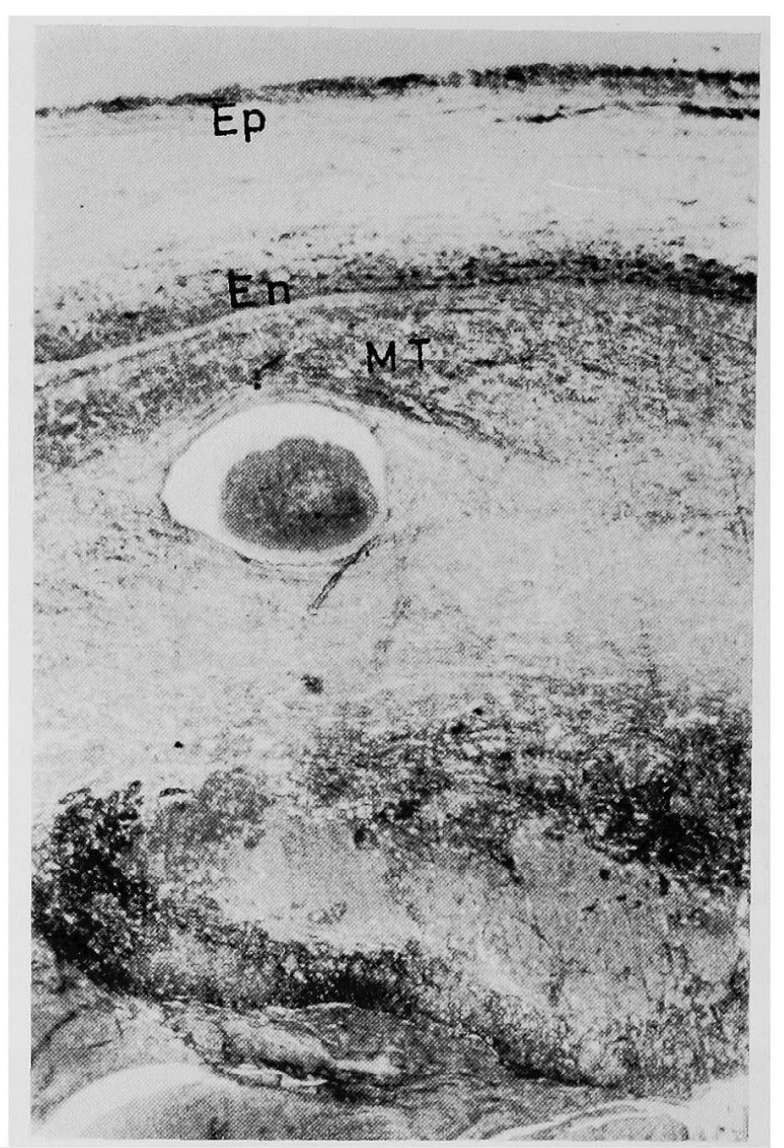

Fig. 5. Microscopic section from the free wall of the right ventricle.

The myocardium is totally absent in this section and the free wall of the right ventricle is mainly composed of fatty tissue. (Mallory's collagen stain, $\times 20$. Ep: epicardium, En: endocardium, MT: mural thrombus).

The right ventricle was fibrotic and paper thin as seen in Fig. 4. The myocardium of the free wall of the right ventricle was almost totally absent as seen in Fig. 5 except for localized area of outflow tract. Even the thickest part of the free wall of the right ventricle measured less than $1 \mathrm{~mm}$. The papillary muscles in the right ventricle were moderately fibrotic. Although the tricuspid valve ring was tremendously dilated, the valve was normally situated. The left ventricle was moderately dilated as seen in Fig. 6 and its thickest part measured $7 \mathrm{~mm}$. There was marked myocardial fibrosis of left ventricle especially in the subepicardial layer as seen in Fig. 7. The pulmonary, mitral and aortic valves were normal. At histological examination, inflammatory cell infiltrations were not demonstrated. The endocardium of the both ventricles was slightly fibrotic. 


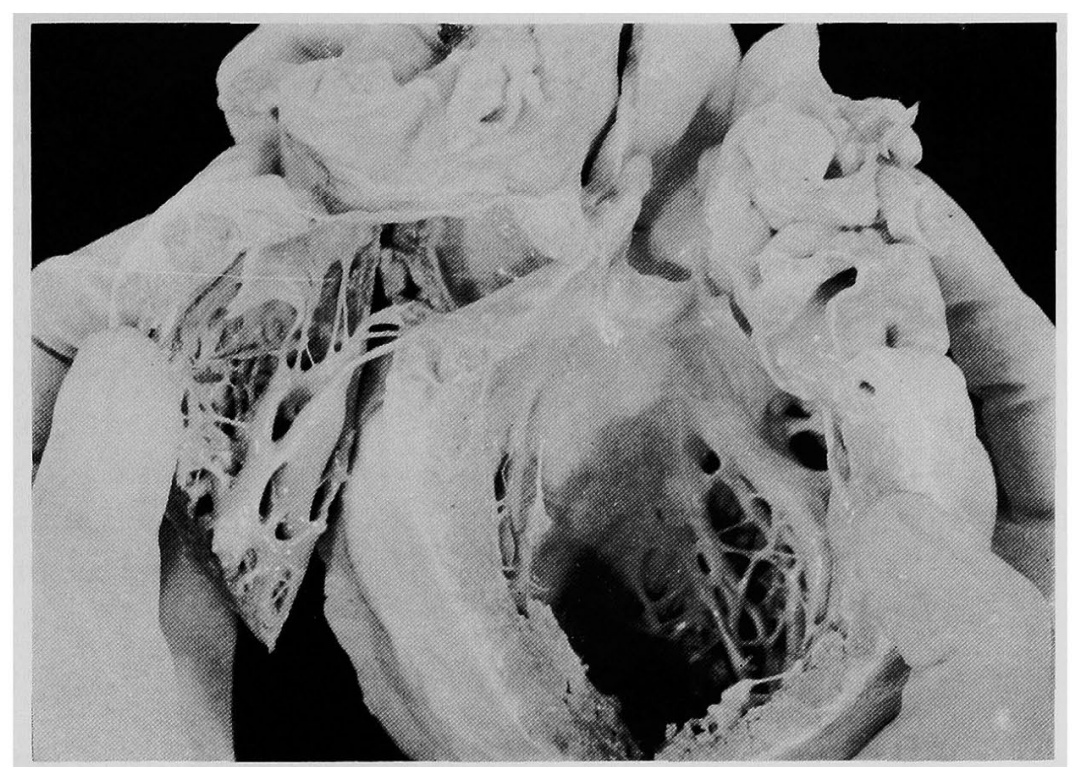

Fig. 6. Opened left ventricle.

Extensive fibrotic change is observed in the subepicardial layer.

\section{Discussion}

The most striking finding of the present case at autopsy was the enormous dilatation of the right atrium and right ventricle with almost total absence of the myocardium of the free wall of the right ventricle. The above findings closely resembled Uhl's anomaly, but there was also a moderate dilatation of the left ventricle associated with marked myocardial fibrosis, especially in the subepicardial layer.

Although the present case may be considered to be a variant form of Uhl's anomaly, we thought it would be better to diagnose as " an unusual type of idiopathic cardiomyopathy of congestive type", because the associated myocardial fibrosis of the left ventricle was quite prominent. The characteristic pathological finding of Uhl's anomaly hitherto described is the almost total absence of the myocardium of the right ventricle without pathological changes in the left heart. ${ }^{1,2)}$ On the basis of the pathological findings, Uhl postulated that the primary developmental defect might be the most likely explanation for the pathogenesis of this anomaly, although possibilities were also raised that the lesion was the result of myocardial infarction with healing or a defect from intrauterine destruction of the myocardium by an inflammatory or degenerative process. ${ }^{1)}$ A possibility of healed myocardial infarction could hardly be considered in the present case, while the primary developmental 


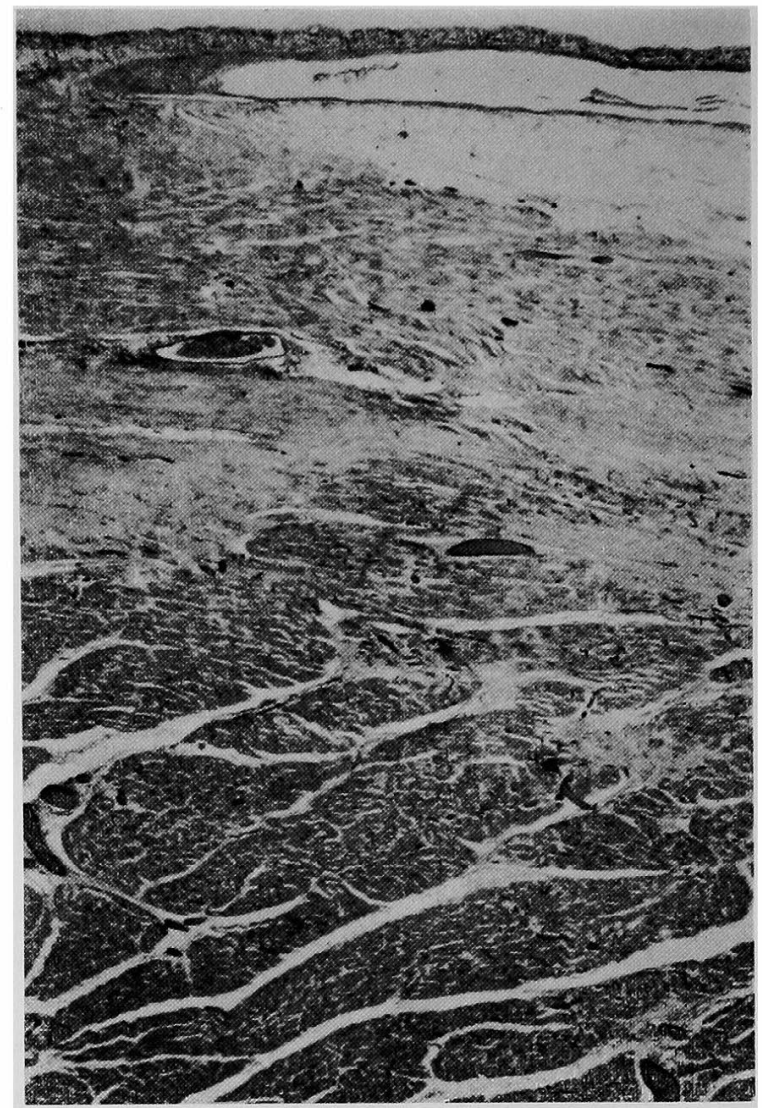

Fig. 7. Microscopic section of the free wall of the left ventricle showing extensive fibrosis in subepicardial layer. (Hematoxylin and eosin, $\times 20$ ).

defect of the right ventricle also seems to be an unlikely explanation because extensive myocardial fibrosis of the left ventricle was associated. Among hitherto reported cases of Uhl's anomaly, at least 2 were associated with pathological change of the left ventricle. Arcilla and Gasul ${ }^{4)}$ reported a 5-monthold male with paper thin right ventricle associated with hypertrophy and dilatation of the left ventricle, while Kuribayashi and associates ${ }^{5)}$ in Japan reported a 17-year-old female diagnosed as Uhl's anomaly with obvious myocardial scars in the left ventricle. Although the myocardial fibrosis of the left ventricle was more extensive in the present case, the case reported by Kuribayashi and associates resembles ours and we are not convinced whether it is appropriate to diagnose their case as "Uhl's anomaly". It was also quite peculiar in the present case that the myocardial fibrosis of the left ventricle was very prominent in the subepicardial layer.

It is noteworthy to point out the unusual pressure tracing curves recorded 
during the right heart catheterization. The pattern of the pressure tracing curve in the right ventricle was virtually the same as in the right atrium, and therefore the position of the catheter could not be precisely determined without help of intracavitary electrocardiogram. There was no evidence of systolic contraction of the right ventricle. A similar observation has been reported by Tomita and associates ${ }^{3)}$ and Kuribayashi and associates ${ }^{5}$ in Uhl's anomaly, whereas Arcilla and Gasul ${ }^{4)}$ described that a systolic contraction of the right ventricle still occurred in Uhl's anomaly in spite of an almost total absence of the myocardium of the right ventricle. In the present case, a possibility of Ebstein's anomally was seriousy considered at the catheter laboratory before the observation of right ventriculography, which revealed the normal situation of the tricuspid valve.

\section{REFERENCES}

1. Uhl HSM: A previously undescribed congenital malformation of the heart: almost total absence of the myocardium of the right ventricle. Bull Johns Hopkins Hosp 91: 197, 1952

2. Ostermeyer J: Uhl's disease: partial parchment right ventricle. Virchows Arch Path Anat Histol 362: 185, 1974

3. Tonita T, et al: A case of hypoplasia of the myocardium of the right ventricle. Shinzo 4: 1372, 1972 (in Japanese)

4. Arcilla RA, Gasul BM: Congenital aplasia or marked hypoplasia of the myocardium of the right ventricle (Uhl's anomaly). Clinical, angiographic, and hemodynamic findings. J Pediatrics 58: 381, 1961

5. Kuribayashi $Y$, et al: A case of hypoplasia of myocardium of right ventricle. Shinzo 4:237, 1972 (in Japanese) 